This is the author's final, peer-reviewed manuscript as accepted for publication. The publisher-formatted version may be available through the publisher's web site or your institution's library.

\title{
Intervertebral disc regeneration: Influence of growth factors on differentiation of human mesenchymal stem cells (hMSC)
}

Franziska Ehlicke, Denise Freimark, Birthe Heil, Adriaan Dorresteijn, Peter Czermak

How to cite this manuscript (APA format)

If you make reference to this version of the manuscript, use the following citation format:

Ehlicke, F., Freimark, D., Heil, B., Dorresteijn, A., Czermak, P. (2010). Intervertebral disc regeneration: Influence of growth factors on differentiation of human mesenchymal stem cells (hMSC). Retrieved from http://krex.ksu.edu

\section{Published Version Information}

Citation: Ehlicke, F., Freimark, D., Heil, B., Dorresteijn, A., Czermak, P. (2010). Intervertebral disc regeneration: Influence of growth factors on differentiation of human mesenchymal stem cells (hMSC). International Journal of Artificial Organs, 33 (4), 244252.

Copyright: The International Journal of Artificial Organs is published and copyrighted by Wichtig Editore - Milano (Italy)

Digital Object Identifier (DOI):

Publisher's Link: http://www.artificialorgans.com/public/IJAO/Article/Articleabstract.aspx?UidArticle=BF0B8C5F-8D9B-4494AF30-439D44DC1C70\&t=IJAO

This item was retrieved from the K-State Research Exchange (K-REx), the institutional repository of Kansas State University. K-REx is available at http://krex.ksu.edu 
Title: Intervertebral Disc Regeneration: Influence of growth factors on differentiation of human mesenchymal stem cells (hMSC)

Running head: Cell-based Intervertebral Disc Regeneration

Franziska Ehlicke ${ }^{1}$, Denise Freimark ${ }^{1}$, Birthe Heil ${ }^{1}$, Adriaan Dorresteijn ${ }^{2}$, Peter Czermak $^{1,3}$

${ }^{1}$ Institute of Biopharmaceutical Technology, University of Applied Sciences GiessenFriedberg, Giessen, Germany

${ }^{2}$ Institute of Zoology, University of Giessen, Germany

${ }^{3}$ Department of Chemical Engineering, Kansas State University, Manhattan KS, USA

Corresponding author: Franziska Ehlicke, Peter Czermak, Wiesenstrasse 14, 35390 Giessen, Germany, phone +496413092630, fax +496413092553, e-mail franziska.ehlicke@tg.fh-giessen.de, peter.czermak@tg.fh-giessen.de

Disclaimers:

The authors are financially supported by the research funding program "LOEWE Landesoffensive zur Entwicklung wissenschaftlich-ökonomischer Exzellenz" of Hessen's Ministry of Higher Education, Research, and the Arts.

The authors declare no conflict of interest.

\section{ABSTRACT}


Introduction: One common cause of disability in modern society is low back pain. The main reason for this pain is the degeneration of the intervertebral disc (IVD), particular of the nucleus pulposus (NP). For an early degeneration stage cell-based therapy would be a minimal invasive method of treatment. Therefore, adequate cells are needed. As the usage of NP cells is limited because of their insufficient amount or vitality, a promising alternative is the application of human mesenchymal stem cells (hMSCs). Objective: To investigate the potential of various growth factors to induce the differentiation of hMSCs into NP cells and thereby to obtain an alternative cell source for the treatment of IVD degeneration. Methods: hMSC-TERT were cultivated three-dimensionally in a hydrogel for 21 days to form NP cells. Cell survival and proliferation were determined using SybrGreen/propidium iodide double staining and the WST-test. To investigate the ability of several growth factors to differentiate hMSCs into NP cells, fluorescence immunostaining of NP-specific marker proteins (e.g. chondroadherin (CHAD) and the recently discovered cytokeratin 19 [1]) was performed. Results: Following the procedure described above, cells are able to maintain their viability and proliferation capacity throughout the cultivation time. By using a previously established immunofluorescence protocol, we could indicate the ability of three different growth factors to differentiate hMSCs into NP-like cells. Conclusion: The expression of several marker proteins in all differentiation experiments indicates the ability of IGF-1, FGF-2 and PDGF-BB to differentiate hMSCs into NP-like cells apart from the usually applied TGF- $\beta 3$. Furthermore, our findings preclude the application of Cytokeratin 19 as a specific marker protein for NP cells [1]. Further experiments have to be done to find real specific NP marker proteins to indisputable verify the differentiation of hMSCs into NP cells. If so, application of the mentioned three growth factors would possibly be an option to obtain sufficient NP cells for minimal invasive IVD regeneration.

\section{KEYWORDS}

intervertebral disc regeneration; differentiation; growth factor; IGF-1; FGF-2; PDGF$\mathrm{BB}$ 


\section{INTRODUCTION}

The degeneration of the intervertebral disc (IVD), particular of the nucleus pulposus (NP), is one reason for low back pain, which is a common disease in Western societies. The major reason for this pain is the pressing of the NP from the inside of the IVD towards the anulus fibrosus on the outside of the disc and finally towards the spinal cord. IVD degeneration progresses in stages, starting with an increasing damage of the NP which leads to the mentioned bulging (IVD protrusion). As a consequence, the anulus fibrosus ruptures and thus, the nucleus pulposus leaks into the spinal canal (IVD prolaps). In the stage of IVD protrusion where the anulus fibrosus is (almost) intact, one possible method of treatment is a cell-based therapy of the NP. Therefore, adequate cells e.g. NP cells are needed. One major problem in using these cells is their insufficient amount and vitality resulting from the progressing IVD degeneration. Moreover, an auxiliary operation is necessary to extract autologous NP cells. In contrast to NP cells, vital human mesenchymal stem cells (hMSC) are available in sufficient amount in tissue. Besides the immune privilege of the avascular structured IVD itself, hMSCs avoid allorecognition and, therefore, circumvent immune response [2,3]. For these reasons our workgroup focuses on the application of hMSCs for IVD regeneration.

hMSCs must be differentiated into NP cells prior to implementation into the body. A three-dimensional environment is required as NP cells dedifferentiate when growing in a monolayer $[4,5]$. Currently, NP cells and the differentiation process are poorly understood. This results from the presumption that NP cells are considered to be chondrocytes. This seems to be not correct since the gene expression profile of IVD cells significantly differs from that of chondrocytes [6]. Our workgroup focuses on the distinction between NP cells (e.g. to NP cells differentiated hMSCs), undifferentiated stem cells and chondrocytes by using fluorescence immunostaining. Based on the results from Steck et al. [6] cartilage oligomeric matrix protein (COMP), chondroadherin (CHAD) and collagen II were chosen as marker proteins to discriminate between undifferentiated hMSCs, chondrocytes and IVD cells in general. Additionally, cytokeratin 19 (Krt 19) was selected as a putative NP-specific marker protein because its mRNA expression has been shown to be significantly higher in NP cells compared to anulus fibrosus and articular cartilage cells [1]. 
Apart from the three-dimensional environment, various stimuli are essential for the differentiation of stem cells. In vivo, growth factors and the extracellular matrix form cellular niches to regulate the proliferation and differentiation of the embedded cells [7]. Therefore, our workgroup started with the investigation of growth factors regarding their applicability to differentiate hMSCs into NP cells. Usually, transforming growth factor- $\beta 3$ (TGF- $\beta 3$ ) is used to induce the differentiation of mesenchymal stem cells into NP-like cells [6, 8-10]. Unfortunately, hMSCs with TGFB3 induced differentiation showed expression of genes typically for hypertrophic chondrocytes or bone [6]. For subsequent therapeutical use of the differentiated cells for IVD regeneration, only original NP cells (e.g. to NP cells differentiated stem cells) can be used. Therefore, it is necessary to find alternate growth factors which stimulate stem cells only into the lineage of NP cells. Pratsinis and Kletsas [11] described insulin-like growth factor 1 (IGF-1), fibroblast growth factor 2 (FGF-2) and platelet-derived growth factor BB (PDGF-BB) as growth factors which are typically expressed in the IVD of various species. These three growth factors have been shown to induce the proliferation of NP and AF cells [11]. The aim of our working group was to find out whether these three growth factors have additional differentiation inducing capabilities. If so, their application instead of TGF- $\beta 3$ might open up a possible way to obtain sufficient NP cells for IVD regeneration.

\section{MATERIAL AND METHODS}

\section{Cell cultivation}

Human mesenchymal stem cells with reverse telomerase transcriptase (hMSCTERT) were obtained from CellMed, Alzenau, Germany. This genetic modification allows an increasing generation number because the telomeres aren't shortened during mitosis. For preliminary cell expansion, Eagle's minimal essential medium (EMEM; PAA, Pasching, Austria) supplemented with 10\% fetal bovine serum (FBS; PAA), $100 \mathrm{U} / \mathrm{ml}$ Penicillin and $100 \mu \mathrm{g} / \mathrm{ml}$ Streptomycin (both Biochrom, Berlin, Germany) was used. The cells were cultivated in a humidified incubator at $37^{\circ} \mathrm{C}$ with $5 \% \mathrm{CO}_{2}$.

Human nucleus pulposus cells (HNPC) were obtained from ScienCell (Carlsbad, USA) and cultured in nucleus pulposus cell medium (ScienCell) with supplements provided by the manufacturer. The cells were cultivated in a humidified incubator at $37^{\circ} \mathrm{C}$ with $5 \% \mathrm{CO}_{2}$. 


\section{Cell differentiation}

Dulbecco's modified Eagle's medium (DMEM-LG; Biochrom) containing 3.7 mg/ml sodium hydrogen carbonate (Carl Roth, Karlsruhe, Germany), $100 \mathrm{U} / \mathrm{ml}$ Penicillin and $100 \mu \mathrm{g} / \mathrm{ml}$ Streptomycin (both Biochrom), 1x Insulin-Transferrin-Selenium-X (Invitrogen, Germany), $0.5 \mathrm{mg} / \mathrm{ml}$ bovine serum albumin (Carl Roth), $1.25 \mathrm{mg} / \mathrm{ml}$ human serum albumin (Sigma-Aldrich, Steinheim, Germany), $4.7 \mu \mathrm{g} / \mathrm{ml}$ linolic acid (AppliChem, Darmstadt, Germany), $0.1 \mu \mathrm{M}$ dexamethasone (Sigma-Aldrich), $0.1 \mathrm{mM}$ 2-phospho-L-ascorbic acid trisodium salt (Sigma-Aldrich) and $0.28 \mathrm{nM}$ growth factor was used for differentiation of hMSC-TERT into NP cells. Depending on the experiment, human Insulin-like Growth Factor-1 (IGF-1; Sigma-Aldrich), human Fibroblast Growth Factor-basic (FGF-2; Biochrom), human Platelet Derived Growth Factor-BB (PDGF-BB; Biochrom) or Transforming Growth Factor- $\beta 3$ (TGF-ß3; Biochrom), were added to the differentiation media.

\section{Cell differentiation by using cell pellets}

To evaluate the differentiation and immunostaining protocol, preliminary differentiation was done in form of pellet cultures with the known differentiation inducing growth factor TGF- $\beta 3$. Therefore, $2 \cdot 10^{5}$ hMSC-TERT were washed with $2.5 \mathrm{ml}$ differentiation medium without TGF- $\beta 3$, centrifuged (5 $\mathrm{min}$ at $150 \mathrm{~g}$ ), resuspended in differentiation medium which included TGF- $\beta 3$ and once again centrifuged (5 min at $150 \mathrm{~g}$ ). Medium was exchanged every 2-3 days. On cultivation days $1,4,7,11,16$ and 21 , cell pellets were fixed with $4 \%$ paraformaldehyde for 20 min at room temperature and stored in phosphate buffered saline (PBS; Biochrom) at $4^{\circ} \mathrm{C}$ until immunostaining.

\section{Cell differentiation in hydrogels}

Subsequent differentiation of hMSC-TERT was done in hydrogels of $2 \%$ low gellingpoint agarose (Sigma-Aldrich) for 21 days to form NP cells. $2.4 \cdot 10^{5}$ cells per $600 \mu \mathrm{l}$ hydrogel were used. Differentiation was done in 12 well-plates at $37{ }^{\circ} \mathrm{C}$ and $5 \% \mathrm{CO}_{2}$ with media exchange every 3-4 days. By addition of IGF-1, FGF-2 or PDGF-BB to the differentiation medium (without TGF- $\beta 3$ ), the ability of these growth factors to support the differentiation of hMSCs into NP cells, was tested. On cultivation days $1,4,7,11$, 
15 and 21, the hydrogels were fixed with 4\% paraformaldehyde (in PBS; Biochrom) for 20 min at room temperature and stored in PBS at $4^{\circ} \mathrm{C}$ until immunostaining.

\section{Measurement of cell viability}

Cell survival was estimated by SybrGreen (1/25 in dimethyl sulfoxide; Sigma-Aldrich) and propidium iodide (5 g/l in PBS; Sigma-Aldrich) staining as described in [12]. Livedead-ratio of cells was analyzed using fluorescence microscopy.

\section{Measurement of cell proliferation}

Cell proliferation reagent WST-1 (Roche Diagnostics, Mannheim, Germany) was used for quantification of the metabolic activity (cell respiration) of the cells within the hydrogels at different time points during the cultivation. On days $0,3,6,10,14$ and $20,20 \mu \mathrm{l}$ of WST-1 were added to $1,980 \mu \mathrm{l}$ of fresh differentiation medium and incubated in a humidified incubator at $37^{\circ} \mathrm{C}$ for $19 \mathrm{~h}$ and $45 \mathrm{~min}$. Medium with WST1 alone served as negative control. Absorbance was measured at $450 \mathrm{~nm}$ with a microplate reader (Synergy HT, BioTek, Bad Friedrichshall, Germany). Information about cell proliferation was obtained by comparison of the absorbance at different time points.

\section{Immunostaining of specific marker proteins}

NP-specific marker proteins (cartilage oligomeric matrix protein (COMP), chondroadherin (CHAD), collagen II and cytokeratin 19 (Krt 19)) were stained by immunofluorescence in hydrogels, fixed on cultivation day $1,4,7,11,15$ or 21 . As blocking solution, 0.01 M PBS (pH 7.2; Biochrom) with 2\% serum (species same as secondary antibody; PAA), 1\% BSA (Carl Roth), 0.1\% Triton X-100 (Carl Roth) and 0.05\% Tween 20 (Carl Roth) was used. After 1 hour incubation at room temperature, the blocking solution was discarded and $2 \mu \mathrm{g} / \mathrm{ml}$ primary antibodies (mouse antihuman COMP: Kamiya Biomedical Company, MC-810; mouse anti-human CHAD: Abnova, H00001101-M01; rabbit anti-human collagen II: Biologo, CO20211-0.1; mouse anti-human cytokeratin 19: AbD Serotec, 5552-9009) were added in primary antibody dilution buffer (0.01 M PBS, 1\% BSA, 0.5\% Triton X-100, pH 7.2). After 1 hour incubation at room temperature, the hydrogels were washed three times with washing solution (0.01 M PBS, 0.05\% Tween 20, pH 7.2). Thereafter, secondary antibodies (6 $\mu \mathrm{g} / \mathrm{ml}$ sheep anti-rabbit FITC: Yo Proteins AB, 173; $10 \mu \mathrm{g} / \mathrm{ml}$ goat anti- 
mouse TR: Fitzgerald Industries International, 43C-CB5102) were added. After 30 minutes of incubation at room temperature and following three wash steps, the hydrogels were counterstained with DAPI (AppliChem, Germany) for 20 minutes at room temperature. After three concluding washing steps, the hydrogels were ready for analysis via fluorescence microscopy.

\section{Fluorescence microscopy}

Immunostained or SybrGreen/propidium iodide stained hydrogels were transferred into a multiwell plate with glass bottom and kept in PBS to prevent dehydration and analyzed via a fluorescence wide-field microscope (DMI6000, Leica Microsystems, Wetzlar, Germany) using filter cubes A4 (ex.: BP 360/40, em.: BP 470/40), L5 (ex.: BP 480/40, em.: BP 527/30) and N3 (ex.: BP 546/12, em.: BP 600/40) and LAS AF Software (Leica Microsystems).

\section{RESULTS}

Using immunofluorescence staining we could detect cartilage oligomeric matrix protein (COMP), chondroadherin (CHAD) and collagen II in hMSC-TERT cell pellets at specific time points during the 21 days of differentiation with TGF- $\beta 3$ (Fig. 1).

Conventional pellet cultivation contrasts with the low cell density of NP cells in vivo. Therefore, we changed to differentiation within agarose hydrogels. Using SybrGreen/ propidium iodide staining we investigated the survival of hMSC-TERT after the whole differentiation period at different cell densities in order to obtain the most suitable. Independent of the density, cells remain viable throughout the whole cultivation time (data not shown). Because of the good live-dead-ratio of cells we selected 2.4.105 cells per hydrogel (Fig. 2) for experiments to evaluate the influence of growth factors on differentiation of hMSC-TERT into NP cells.

Within these hydrogels, cell proliferation throughout the 21 days of differentiation was quantified using the WST-test for measurement of cell respiration (more precisely the activities of several dehydrogenase) (Fig. 3). All four growth factor approaches showed different behavior in cell proliferation during the 21 days of differentiation. In contrast to TGF- $\beta 3$ and IGF-1, which acted similarly with highest cell respiration activity on day 7 and 15, FGF-2 led to highest cell respiration activity on day 7 and showed a subsequent decrease. With the use of PDGF-BB, highest cell respiration activity was detected over a time period from day 7 to day 15 . 
Based on the results from the staining in cell pellets (Fig.1), adequate time points during differentiation were chosen for fluorescence immunostaining of the different marker proteins (COMP, CHAD and collagen II). Fluorescence immunostaining of the hydrogels revealed high expression of collagen II but low expressions of COMP and CHAD within all four differentiation approaches (Fig. 4). Expression of cytokeratin 19 (Krt 19) was detected throughout the whole differentiation time with comparatively high expression in the approach with IGF-1 (Fig. 5). Actually, Krt 19 expression decreased with increasing differentiation time (Fig. 5). Nevertheless, human nucleus pulposus cells cultivated for 23 days in a hydrogel showed expression of Krt 19 as well (Fig. 6).

\section{DISCUSSION}

In this study, we used hMSC-TERT instead of primary hMSCs not only to achieve increased generation numbers but also to obtain a standardized cell line without the differences usually observed when various donor sources are used.

hMSC-TERT remain viable throughout the whole cultivation time when cultured in hydrogels of $2 \%$ agarose in differentiation medium which included TGF- $\beta 3$. Because of the good live-dead-ratio of cells, $2.4 \cdot 10^{5}$ cells per $600 \mu$ hydrogel were chosen for the investigations concerning the putative differentiation potential of IGF-1, FGF-2 and PDGF-BB. Our working group assumed that hMSC-TERT cultivated in differentiation inducing media with IGF-1, FGF-2 and PDGF-BB will survive, too. A density of $2.4 \cdot 10^{5}$ cells per $600 \mu$ hydrogel conforms to a cell density of $4 \cdot 10^{5}$ cells $/ \mathrm{cm}^{3}$. As shown in this study, for this cell concentration, $0.28 \mathrm{nM}$ of the three tested growth factors are able to stimulate the differentiation of human mesenchymal stem cells into NP-like cells. In vivo, NP cells exist in concentrations of about $4 \cdot 10^{6}$ cells $/ \mathrm{cm}^{3}$ [13]. In combination, $2.8 \mathrm{nM}$ of the mentioned growth factors would be able to induce differentiation of hMSC-TERT into NP-like cells when cultured under in vivolike conditions. As cell-cell-interactions by means of cellular crosstalk may also contribute to differentiation processes, the experiments described here have to be repeated with the natural NP cell-concentration.

This study shows that all three growth factors (IGF-1, FGF-2 and PDGF-BB) can stimulate the differentiation of hMSC-TERT into the lineage of NP cells. According to our current knowledge, no investigations have been performed yet which show the differentiation of hMSCs into NP-like cells by means of growth factors (except for 
TGF- $\beta 3$ with the resulting drawbacks mentioned in the introduction). However, regarding osteogenic and chondrogenic differentiation, FGF-2 has been shown to increase the differentiation potentials of hMSCs when added to the culture media before the differentiation was started by changing to specific differentiation-inducing medium [14]. It has been shown that the applied growth factors can stimulate the proliferation of NP cells. Pratsinis and Kletsas determined the concentrations of IGF-1, FGF-2 and PDGF-BB, which had a maximal stimulatory effect on DNA synthesis as $100 \mathrm{ng} / \mathrm{ml}(13,08 \mathrm{nM}), 5 \mathrm{ng} / \mathrm{ml}(0,30 \mathrm{nM})$ and $10 \mathrm{ng} / \mathrm{ml}(0,41 \mathrm{nM})$ [11]. Zhang et al. described $100 \mathrm{ng} / \mathrm{ml}$ IGF-1 to provoke maximal proliferation of human NP cells in a monolayer as well [15]. It should be mentioned that the experimental conditions of these studies differed from those of our study. Pratsinis and Kletsas used bovine coccygeal NP cells and Zhang et al. human NP cells which were cultured in a monolayer [11, 15], whereas our group used differentiated hMSCs which were cultured three dimensionally in a hydrogel. It is still difficult to compare these studies not only because of the different cell types but also because of the different cultivation settings (monolayer vs. 3D). Especially for NP cells, it is well known that the behavior of cells differs when cultured in a monolayer instead of a three dimensional cultivation [16]. Nevertheless, our investigations show similar results compared to the work of Pratsinis and Klestas [11] because a maximal proliferation stimulation effect could be detected with PDGF-BB in both studies.

In our study with IGF-1, FGF-2 and PDGF-BB, a connection between the proliferation profile and the expression profile of the differentiation markers could not be seen. Surprisingly, almost no cell proliferation could be detected on the last cultivation day even though live-dead-staining revealed almost 95\% cell viability after 21 days of cultivation. One possible explanation could be some limitations within the hydrogel between the cultivation days 15 and 21 which led to cell starvation but not to cell dead. Since live-dead-staining was exemplary performed with TGF- $\beta 3$ as differentiation inducing growth factor, these measurements have to be also done with IGF-1, FGF-2 and PDGF-BB. A negative effect of the used agarose on cell proliferation is unlikely because vital nucleus pulposus cells were successfully cultured within agarose hydrogels [17]. Concerning chondrocytes, agarose could not only be used for cell survival but also for redifferentiation of monolayer cultured cells [18]. Human bone-marrow derived mesenchymal stem cells cultured with chondrogenesis inducing media in $2 \%$ agarose hydrogels survived and expressed 
chondrogenic markers, too [19]. Huang et al. showed a clear relationship between the cell seeding density within the agarose hydrogel and the expression of chondrogenic markers [19]. As highest gene expression could be detected with $9 \cdot 10^{6}$ cells /ml [19], it is likely that the density applied in the present study was too low for optimal cell growth. As mentioned earlier, the growth factor experiments presented in this study will be repeated with the natural occurring NP cell density $\left(4 \cdot 10^{6}\right.$ cells $\left./ \mathrm{ml}\right)$ [13], which will be closer to the cell density from Huang et al. [19]. Therefore, we expect better proliferation and higher expression of the marker proteins within the following study.

Additional experiments could be the analysis of different concentrations or combinations of growth factors to investigate synergistic effects which are known from other cell types as well.

Expressions of collagen II, COMP, CHAD and Krt 19 within the pellets (no Krt 19 staining implemented) and hydrogels proved the applicability of the established fluorescence immunostaining method. The high expression of collagen II for all growth factors investigated was expected as this is the predominant matrix component in the nucleus pulposus [20]. In proportion to the expression of collagen II, few amounts of COMP and CHAD were detected. This correlates with the findings of Steck et al. [6], who assessed the gene expression levels of IVD tissue, cartilage and TGF- $\beta 3$ induced hMSCs. Thereby, it has to be noticed that articular cartilage and anulus fibrosus cells are specified by a significant higher expression of COMP compared to NP cells [1]. Thus, the detected little amount in the study of our working group indicates that the hMSC-TERT cells were differentiated into the lineage of NPlike cells and not into chondrocytes or AF cells. To obtain more indications that hMSC-TERT were differentiated into NP cells we used cytokeratin 19 (Krt 19) as a putative new NP marker protein. In 2009, Rutges et al. compared the mRNA expression profiles of NP cells, anulus fibrosus cells and articular cartilage cells [1]. They found Krt 19 as a putative NP specific marker protein because its mRNA expression has been shown to be significantly higher in NP cells compared to anulus fibrosus and articular cartilage cells [1]. The mRNA expression of Krt 19 was ascertained to be 5 units lower in log-scale compared to mRNA expression of cartilage oligomeric matrix protein (COMP) in NP cells [1]. In contrast to these findings, in our study, expression of Krt 19 was higher than expression of COMP. Furthermore, in the present study, Krt 19 was expressed in all four differentiation 
approaches from the outset of the immunostaining on day 1. Apart from the fact that further experiments have to be done, it is unlikely that first expression of Krt 19 in differentiation into NP cells occurs at day one of cultivation in the medium inducing differentiation. So, Krt 19 seems to be expressed in mesenchymal stem cells, too. Consequently, Krt 19 could not be used for characterizing NP cells if comparisons with hMSCs are required. Nevertheless, expression of Krt 19 was highest in hydrogels cultivated with IGF-1, which could indicate that the differentiation inducing capacity of IGF-1 is higher than the capacities of FGF-2, PDGF-BB and even higher than the capacity of TGF- $\beta 3$.

Further experiments will be done to find real specific NP marker proteins for the discrimination between NP cells, chondrocytes and mesenchymal stem cells.

\section{ACKNOWLEDGEMENT}

We would like to thank the Hessen State Ministry of Higher Education, Research and the Arts for the financial support within the Hessen initiative for scientific and economic excellence (LOEWE-Program).

\section{REFERENCES}

1. Rutges, J., et al., Variations in gene and protein expression in human nucleus pulposus in comparison with annulus fibrosus and cartilage cells: potential associations with aging and degeneration. Osteoarthritis Cartilage, 2009.

2. Liu, H., et al., The immunogenicity and immunomodulatory function of osteogenic cells differentiated from mesenchymal stem cells. J Immunol, 2006. 176(5): p. 2864-71.

3. Ryan, J.M., et al., Mesenchymal stem cells avoid allogeneic rejection. J Inflamm (Lond), 2005. 2: p. 8.

4. Darling, E.M. and K.A. Athanasiou, Rapid phenotypic changes in passaged articular chondrocyte subpopulations. J Orthop Res, 2005. 23(2): p. 425-32.

5. Aulthouse, A.L., et al., Expression of the human chondrocyte phenotype in vitro. In Vitro Cell Dev Biol, 1989. 25(7): p. 659-68.

6. Steck, E., et al., Induction of intervertebral disc-like cells from adult mesenchymal stem cells. Stem Cells, 2005. 23(3): p. 403-11.

7. Schofield, R., The relationship between the spleen colony-forming cell and the haemopoietic stem cell. Blood Cells, 1978. 4(1-2): p. 7-25.

8. Steck, E., et al., Chondrocyte expressed protein-68 (CEP-68), a novel human marker gene for cultured chondrocytes. Biochem J, 2001. 353(Pt 2): p. 169-74.

9. Murdoch, A.D., et al., Chondrogenic differentiation of human bone marrow stem cells in transwell cultures: generation of scaffold-free cartilage. Stem Cells, 2007. 25(11): p. 2786-96.

10. Burdick, J.A. and G. Vunjak-Novakovic, Engineered microenvironments for controlled stem cell differentiation. Tissue Eng Part A, 2009. 15(2): p. 205-19.

11. Pratsinis, $\mathrm{H}$. and D. Kletsas, PDGF, bFGF and IGF-I stimulate the proliferation of intervertebral disc cells in vitro via the activation of the ERK and Akt signaling pathways. Eur Spine J, 2007. 16(11): p. 1858-66. 
12. Barbesti, S., et al., Two and three-color fluorescence flow cytometric analysis of immunoidentified viable bacteria. Cytometry, 2000. 40(3): p. 214-8.

13. Maroudas, A., et al., Factors involved in the nutrition of the human lumbar intervertebral disc: cellularity and diffusion of glucose in vitro. J Anat, 1975. 120(Pt 1): p. 113-30.

14. Ito, T., et al., FGF-2 increases osteogenic and chondrogenic differentiation potentials of human mesenchymal stem cells by inactivation of TGF-beta signaling. Cytotechnology, 2008. 56(1): p. 1-7.

15. Zhang, R., D. Ruan, and C. Zhang, Effects of TGF-beta1 and IGF-1 on proliferation of human nucleus pulposus cells in medium with different serum concentrations. J Orthop Surg Res, 2006. 1: p. 9.

16. Horner, H.A., et al., Cells from different regions of the intervertebral disc: effect of culture system on matrix expression and cell phenotype. Spine (Phila Pa 1976), 2002. 27(10): p. 101828.

17. Shen, B., et al., Induction of matrix metalloproteinase-2 and -3 activity in ovine nucleus pulposus cells grown in three-dimensional agarose gel culture by interleukin-1beta: a potential pathway of disc degeneration. Eur Spine J, 2003. 12(1): p. 66-75.

18. Benya, P.D. and J.D. Shaffer, Dedifferentiated chondrocytes reexpress the differentiated collagen phenotype when cultured in agarose gels. Cell, 1982. 30(1): p. 215-24.

19. Huang, C.Y., et al., Chondrogenesis of human bone marrow-derived mesenchymal stem cells in agarose culture. Anat Rec A Discov Mol Cell Evol Biol, 2004. 278(1): p. 428-36.

20. Eyre, D.R. and H. Muir, Types I and II collagens in intervertebral disc. Interchanging radial distributions in annulus fibrosus. Biochem J, 1976. 157(1): p. 267-70. 
Legends

Figure 1: Immunostaining of NP-specific marker proteins in cell pellets after differentiation with TGF- $\beta 3(0.28 \mathrm{nM})$. As cartilage oligomeric matrix protein (COMP), chondroadherin (CHAD) and collagen II expression started at different time points during differentiation, representative pictures from different fixation days plus positive/negative control were assorted. A: Expression of COMP on cultivation days 0, 4, 7 and 21. B: Expression of CHAD on cultivation days $0,11,16$ and 21 . C: Expression of collagen II on cultivation days 0, 11, 16 and 21. Magnification: 100x.

Figure 2: Cell survival in the hydrogel $(600 \mu \mathrm{l})$ after 21 days of differentiation with TGF- $\beta 3$ (0.28 nM). A: Living cells (green in overlay). B: Dead cells (red in overlay). C: Overlay. Magnification: 100x.

Figure 3: Determination of cell proliferation in dependency on the added growth factor by incubation with WST-1 reagent and measuring the resultant absorbance at $450 \mathrm{~nm}$. Striped: TGF- 33 . White: IGF-1. Grey: FGF-2. Black: PDGF-BB. Bars display mean values $(n=9)$ with standard deviation.

Figure 4.1: Influence of growth factors on the differentiation by immunostaining of NP-specific marker proteins on days 1, 4 and 7. A: cartilage oligomeric matrix protein (COMP; green in overlay). B: DAPI (blue in overlay). C: Overlay. Magnification: 400x

Figure 4.2: Influence of growth factors on the differentiation by immunostaining of NP-specific marker proteins on days 11 and 21. A: chondroadherin (CHAD; red in overlay). B: collagen II (green in overlay). C: DAPI (blue in overlay). D: Overlay. Magnification: 400x

Figure 5: Influence of growth factors on the differentiation by immunostaining of the putative NP-specific marker protein cytokeratin 19 (Krt 19) on days 1, 4, 7, 11, 15 and 21. A: Krt 19 (red in overlay). B: DAPI (blue in overlay). C: Overlay. Magnification: 2,100x (630x optical and $31 / 3 \times$ digital)

Figure 6: Immunostaining of the putative NP-specific marker protein cytokeratin 19 (Krt 19) on NP cells cultivated for 23 days in a hydrogel to sustain the differentiation status. A: Krt 19 (red in overlay). B: DAPI (blue in overlay). C: Overlay. Magnification: 2,100x (630x optical and 3 1/3x digital) 

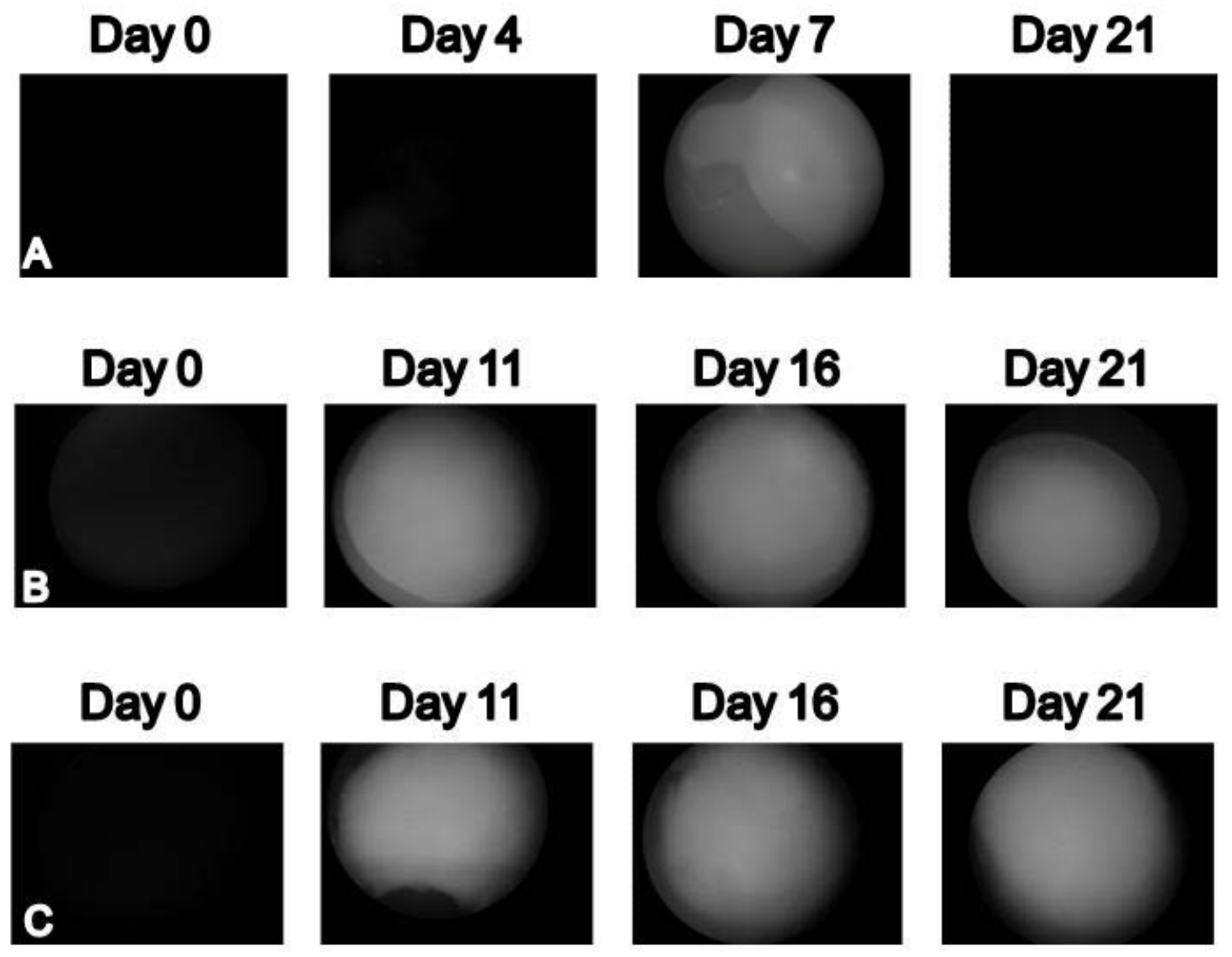

Figure 1

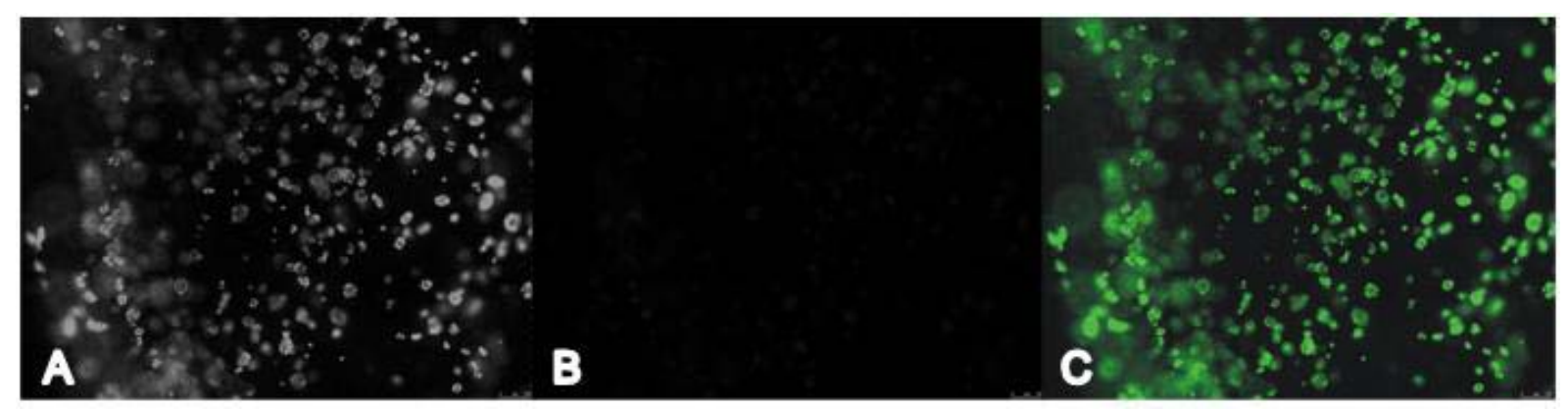

Figure 2 


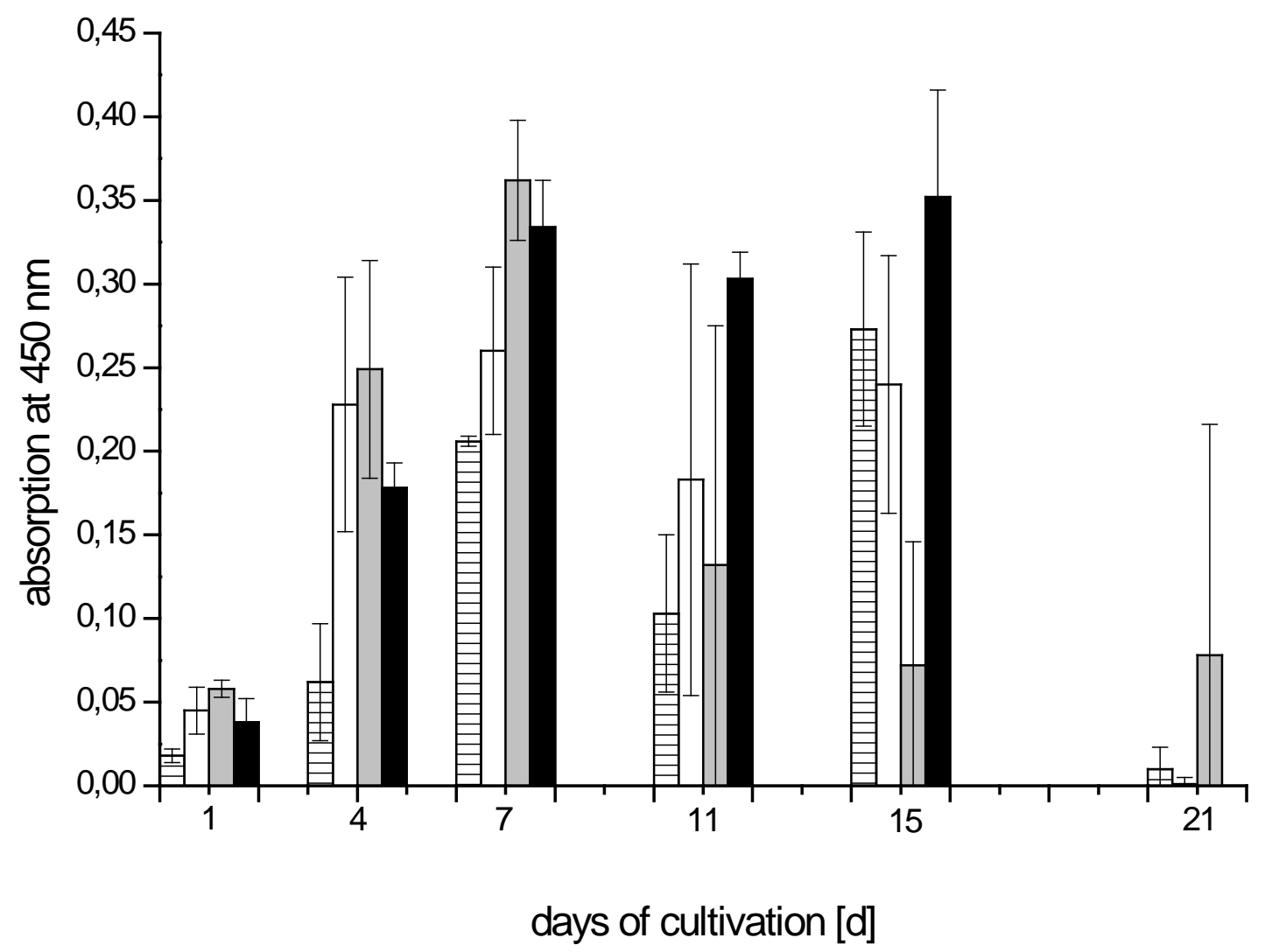

Figure 3 
Day 1
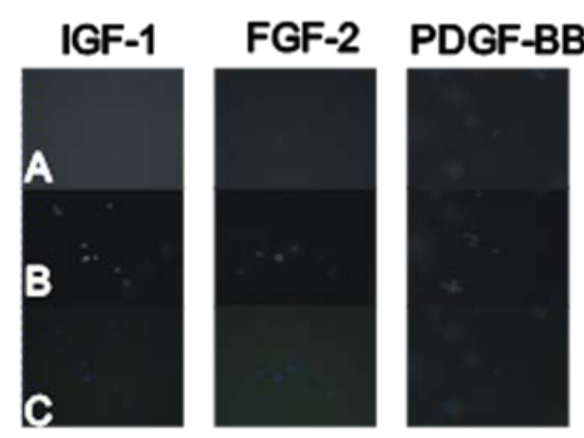

Day 7
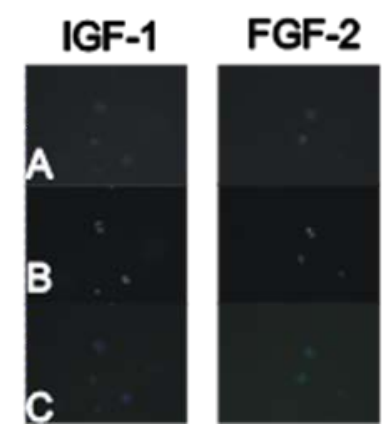

Day 4
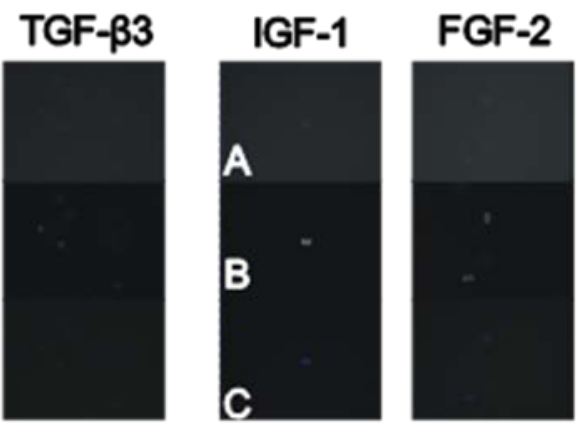
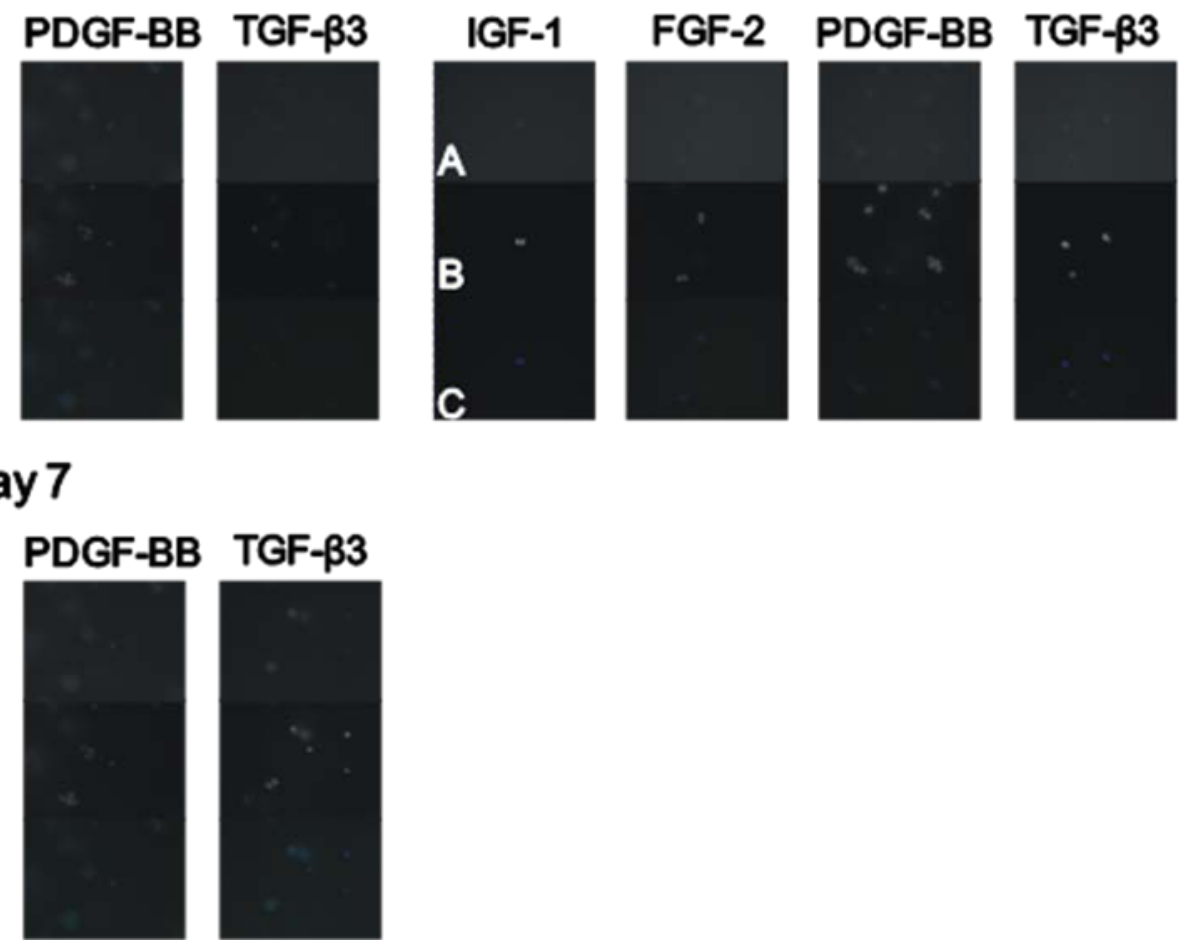

Figure 4.1

Day 11
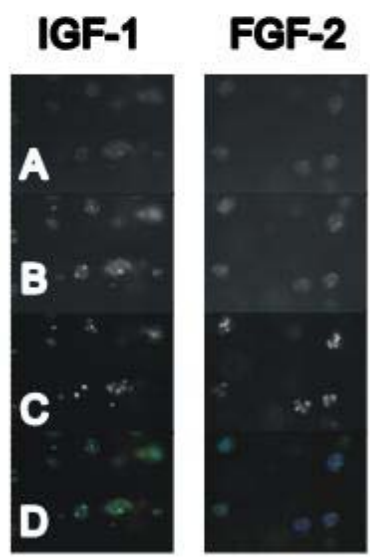
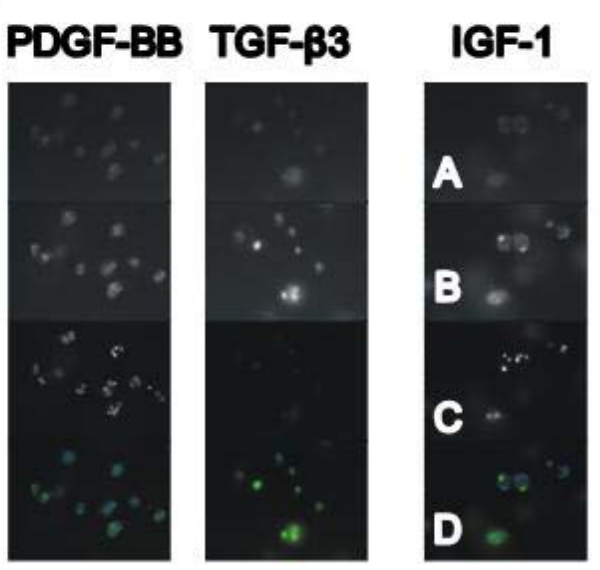

Day 21
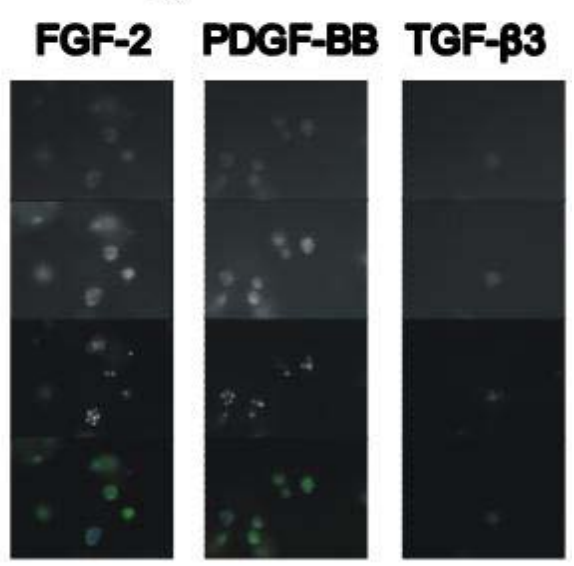

Figure 4.2 
Day 1
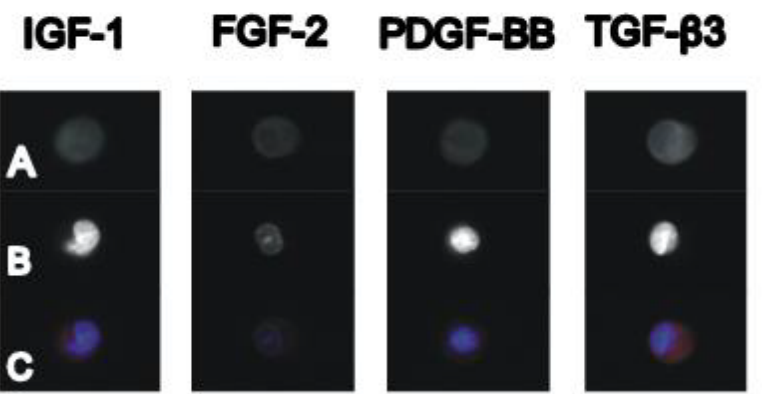

Day 7

IGF-1 FGF-2 PDGF-BB TGF- $\beta 3$
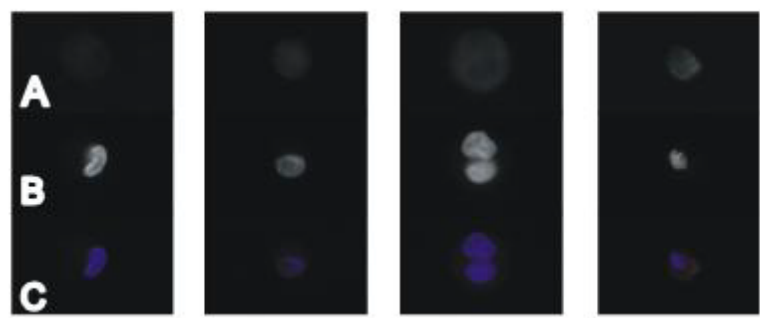

Day 15

IGF-1 FGF-2 PDGF-BB TGF- $\beta 3$
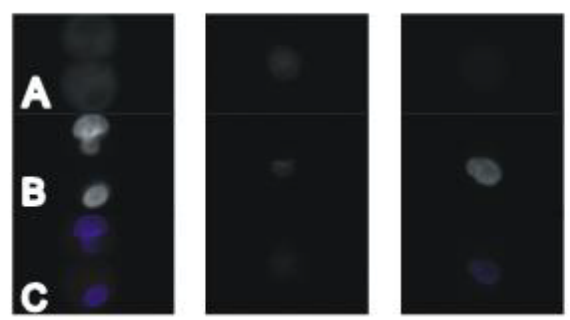

Day 4

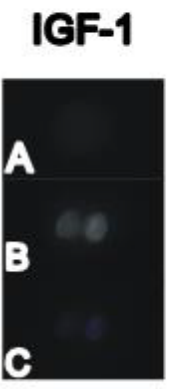

FGF-2
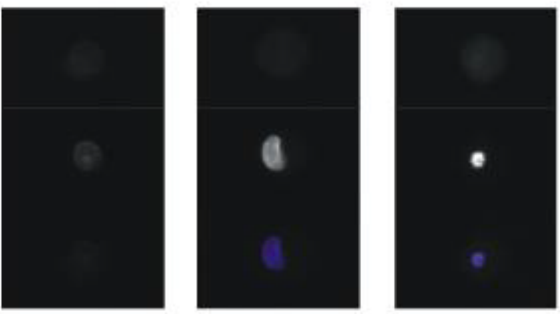

Day 11

IGF-1 FGF-2 PDGF-BB TGF- $\beta 3$
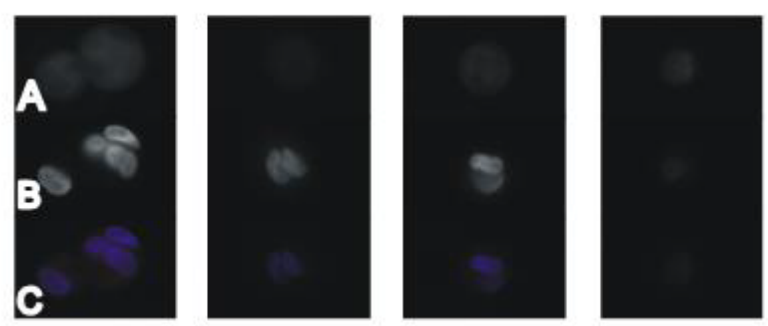

Day 21

IGF-1 FGF-2 PDGF-BB TGF- $\beta 3$
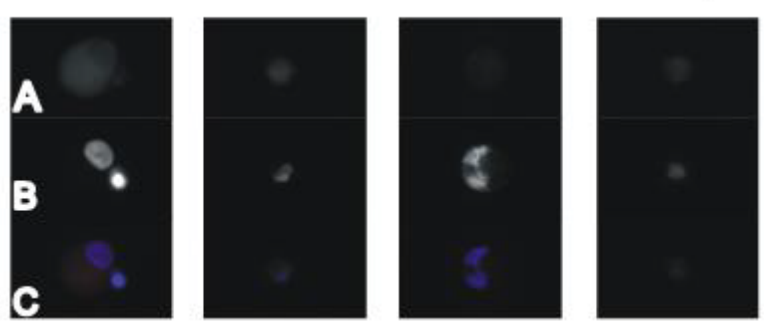

Figure 5

HNPC

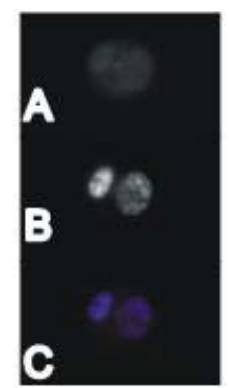

Figure 6 